\title{
New clinical data on human spinal cord re-irradiation tolerance
}

\author{
Hiroshi Doi ${ }^{1,2} \cdot$ Keisuke Tamari ${ }^{1,3} \cdot$ Ryoong-Jin Oh ${ }^{1} \cdot$ Carsten Nieder ${ }^{4,5}$ (D)
}

Received: 16 January 2021 / Accepted: 23 March 2021 / Published online: 5 May 2021

(c) The Author(s) 2021

\begin{abstract}
Purpose To provide additional clinical data about the re-irradiation tolerance of the spinal cord.

Methods This was a retrospective bi-institutional study of patients re-irradiated to the cervical or thoracic spinal cord with minimum follow-up of 6 months. The maximum dose (Dmax) and dose to 0.1cc (D0.1cc) were determined (magnetic resonance imaging $[\mathrm{MRI}]$-defined cord) and expressed as equivalent dose in 2-Gy fractions (EQD2) with an $\alpha / \beta$ value of 2 Gy.

Results All 32 patients remained free from radiation myelopathy after a median follow-up of 12 months. Re-irradiation was performed after 6-97 months (median 15). In 22 cases (69\%) the re-irradiation spinal cord EQD2 Dmax was higher than that of the first treatment course. Forty-eight of 64 treatment courses employed fraction sizes of 2.5 to $4 \mathrm{~Gy}$ to the target volume. The median cumulative spinal cord EQD2 Dmax was 80.7 Gy, minimum 61.12 Gy, maximum $114.79 \mathrm{~Gy}$. The median cumulative spinal cord D0.1cc EQD2 was 76.1 Gy, minimum 61.12 Gy, maximum 95.62 Gy. Besides cumulative dose, other risk factors for myelopathy were present (single-course Dmax EQD2 $\geq 51$ Gy in 9 patients, single-course D0.1cc EQD2 $\geq 51$ Gy in 5 patients).

Conclusion Even patients treated to higher cumulative doses than previously recommended, or at a considerable risk of myelopathy according to a published risk score, remained free from this complication, although one must acknowledge the potential for manifestation of damage in patients currently alive, i.e., still at risk. Individualized decisions to re-irradiate after appropriate informed consent are an acceptable strategy, including scenarios where low re-irradiation doses to the spinal cord would compromise target coverage and tumor control probability to an unacceptable degree.
\end{abstract}

Keywords Spinal metastases $\cdot$ Palliative radiotherapy $\cdot$ Radiation myelopathy $\cdot$ Dose constraint $\cdot$ Treatment planning

\section{Introduction}

Experimental animal data have suggested that spinal cord re-irradiation is a feasible approach $[1,2]$. In clinical practice, several treatment planning and delivery techniques allow for sparing of the spinal cord [3-8]. However, it is not always possible to avoid limited re-irradiation doses, and

\footnotetext{
Hiroshi Doi, MD, PhD

h-doi@med.kindai.ac.jp

Keisuke Tamari, MD, PhD

tamari@radonc.med.osaka-u.ac.jp

Ryoong-Jin Oh, MD

oh@osaka-igrt.or.jp

$\triangle$ Carsten Nieder, MD

cnied@hotmail.com

1 Miyakojima IGRT Clinic, 1-16-22

Miyakojimahondori, Miyakojima-ku, Osaka 534-0021, Japan
}

sometimes high-dose re-irradiation is the only treatment option [9]. A simple method for calculating re-irradiation tolerance is to assume time-dependent recovery (25\% after 6 months, $50 \%$ after 12 months), resulting in tolerance doses of $125 \%$ and $150 \%$, respectively [10]. For example, an initial treatment course that resulted in an equivalent

2 Department of Radiation Oncology, Kindai University Faculty of Medicine, 377-2 Ohno-Higashi, Osaka-Sayama, Osaka 589-8511, Japan

3 Department of Radiation Oncology, Osaka University Graduate School of Medicine, 2-2 Yamadaoka, Suita, Osaka 565-0871, Japan

4 Department of Oncology and Palliative Medicine, Nordland Hospital Trust, 8092 Bod $\varnothing$, Norway

5 Department of Clinical Medicine, Faculty of Health Sciences, UiT-The Arctic University of Norway, Troms $\varnothing$, Norway 
Table 1 Risk factors for radiation myelopathy after re-irradiation

\begin{tabular}{lcl}
\hline Risk factor & Characteristic & Points \\
\hline Time interval & $<6$ months & 4.5 \\
& $\geq 6$ months & 0 \\
EQD2 for first or second course & $\geq 51 \mathrm{~Gy}$ & 4.5 \\
EQD2 for both courses & $<51 \mathrm{~Gy}$ & 0 \\
Cumulative EQD2, both courses & $60.1-65 \mathrm{~Gy}$ & 1 \\
& $65.1-70 \mathrm{~Gy}$ & 2 \\
& $70.1-75 \mathrm{~Gy}$ & 3 \\
& $75.1-80 \mathrm{~Gy}$ & 4 \\
& $80.1-85 \mathrm{~Gy}$ & 5 \\
& $85.1-90 \mathrm{~Gy}$ & 6 \\
\hline
\end{tabular}

$E Q D 2$ equivalent dose in 2-Gy fractions. For doses $>90 \mathrm{~Gy}$ the same principle of adding one point per dose interval applies. Low risk: point sum 0-3, intermediate risk: point sum 4-6, high risk: point sum $>6$.

spinal cord dose (EQD2) close to 50 Gy in 2-Gy fractions may be supplemented by a second course with spinal cord EQD2 of $25 \mathrm{~Gy}$ in 2-Gy fractions 18 months later, i.e., $50 \%$ of the tolerance dose in the first-line setting. For occasional patients, such limited re-irradiation doses are not sufficient to achieve the desired outcome in terms of efficacy or local control [11]. A myelopathy risk score has been developed to inform treatment planning decisions under these special circumstances. The development cohort included 40 individual patients from eight different publications, and 11 of these patients had developed radiation myelopathy [12]. Later, 38 additional patients treated by the authors of that report or published in four different other publications were studied [13]. The risk score based on three variables (cumulative equivalent dose, highest equivalent dose of all treatment series in a particular individual, and time interval between first and second course), which discriminates three different risk groups, did not require modification after evaluation of these 38 new patients. Still $<5 \%$ of the patients in the low-risk group had developed radiation myelopathy. A recent publication from Japan (74 patients) has provided additional clinical data [14]. In the present bi-institutional study, all Japanese data fulfilling certain eligibility criteria were pooled with new data collected by the first author of the risk score in order to confirm the safety of the previous recommendations. Neither institution has encountered any case of re-irradiation myelopathy in the cervical or thoracic spine so far.

\section{Materials and methods}

For the present retrospective study, the Japanese authors provided expanded individual patient data that originated from their previous publication [14]. The Norwegian data, which have not been published previously, were extracted and converted to EQD2 according to the same methods.
The Institutional Review Board at Miyakojima IGRT clinic approved the study. Only patients who were followed for at least 6 months from re-irradiation to the cervical or thoracic spinal cord were eligible. The follow-up information, e.g., clinical symptoms of radiation myelopathy, was obtained from the institutional electronic patient records. Re-irradiation was performed in the time period between 2007 and 2018, with different treatment planning systems and linear accelerators in use. Image guidance, e.g., cone beam computed tomography (CBCT), was not mandatory. Fractionation and total dose were at the discretion of the treating team of radiation oncologists and physicists. Spinal cord EQD2 was calculated according to the linear-quadratic model with an $\alpha / \beta$ value of 2 Gy $[15,16]$, based on the dose-volume histograms of the three-dimensional treatment plans where the true spinal cord had been contoured, as opposed to surrogate structures such as the spinal canal. Both maximum dose (Dmax) and dose to $0.1 \mathrm{cc}(\mathrm{D} 0.1 \mathrm{cc})$ of the re-irradiated spinal cord were tabulated. In case of two-dimensional treatment techniques, spinal cord doses were reconstructed on fused computed tomography (CT) and magnetic resonance imaging (MRI) scans. Coregistered treatment planning scans from the first and second courses were used to assess the cumulative doses in the overlapping region. The risk score was calculated as described previously $[12,13]$ (displayed in Table 1).

\section{Results}

Sixteen adult patients received re-irradiation at the Norwegian center and 16 at the Japanese center. All 32 patients were re-irradiated to the cervical or thoracic spinal cord and were followed for at least 6 months from re-irradiation (median 12 months, maximum 90 months), Table 2 . The median age was 60.5 years. Twenty-four patients (75\%) were re-irradiated for bone metastases (diagnosis code C79.5). Other indications included multiple myeloma, leptomeningeal spinal metastases, and intrathoracic primary tumors, among others. The most common primary tumors were lung cancer (C34.9) in 10 patients (31\%), hepatic cancer $(19 \%)$, and kidney cancer $(12.5 \%)$. Re-irradiation was performed after 6-97 months (median 15). The cervical cord was re-irradiated in 9 patients $(28 \%)$, including those with treatment of the cervicothoracic region such as level C7-Th2. A single vertebra was re-irradiated in 12 patients $(37.5 \%)$, two in 9 patients $(28 \%)$, and three or more in 11 patients $(34 \%)$.

The most common fractionation regimen was 10 fractions of $3 \mathrm{~Gy}$ ( 21 of 64 treatment courses, 33\%). Fortyeight of 64 treatment courses (75\%) employed fraction sizes of 2.5 to $4 \mathrm{~Gy}$ to the target volume. In 22 cases $(69 \%)$, the re-irradiation spinal cord equivalent Dmax was higher 


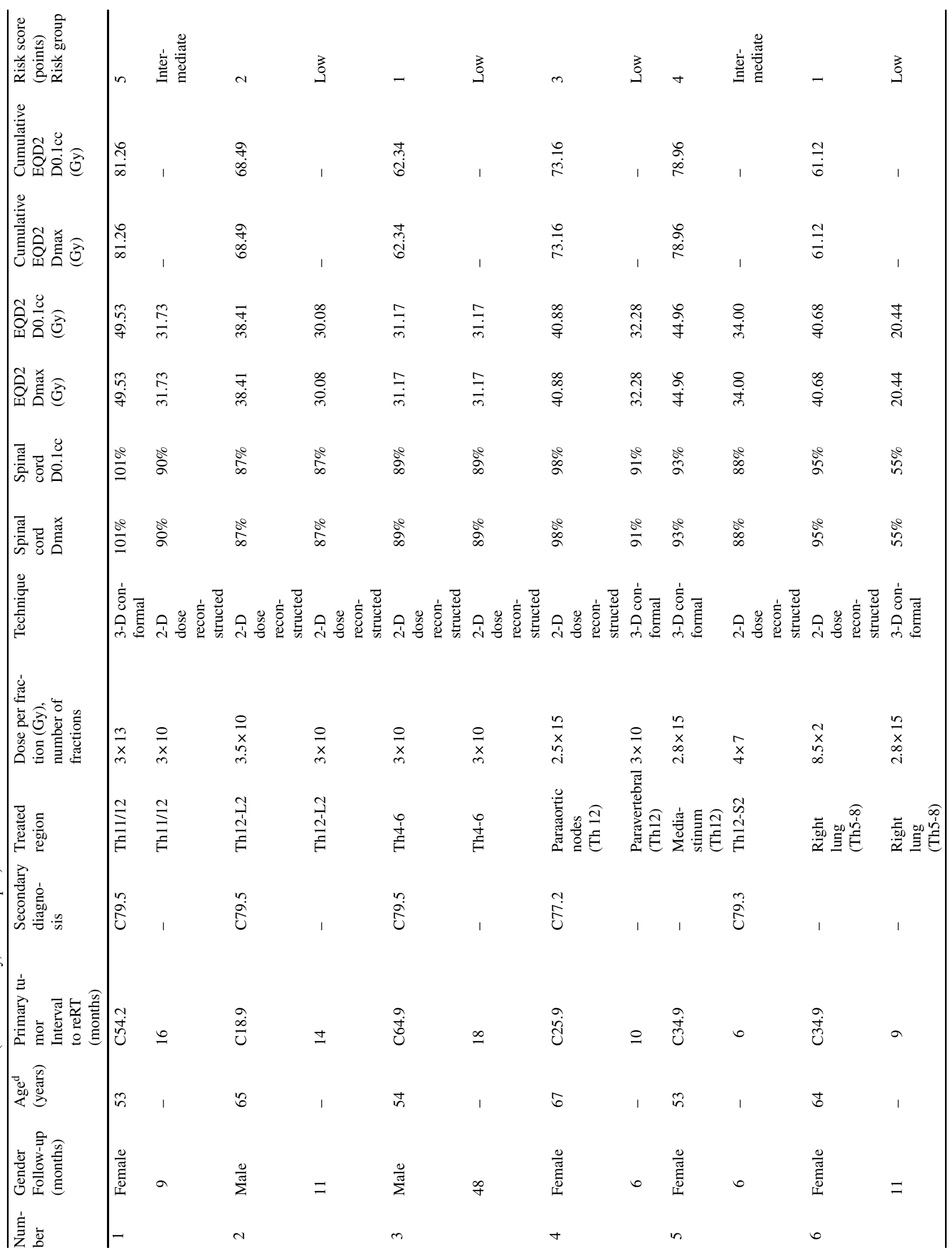




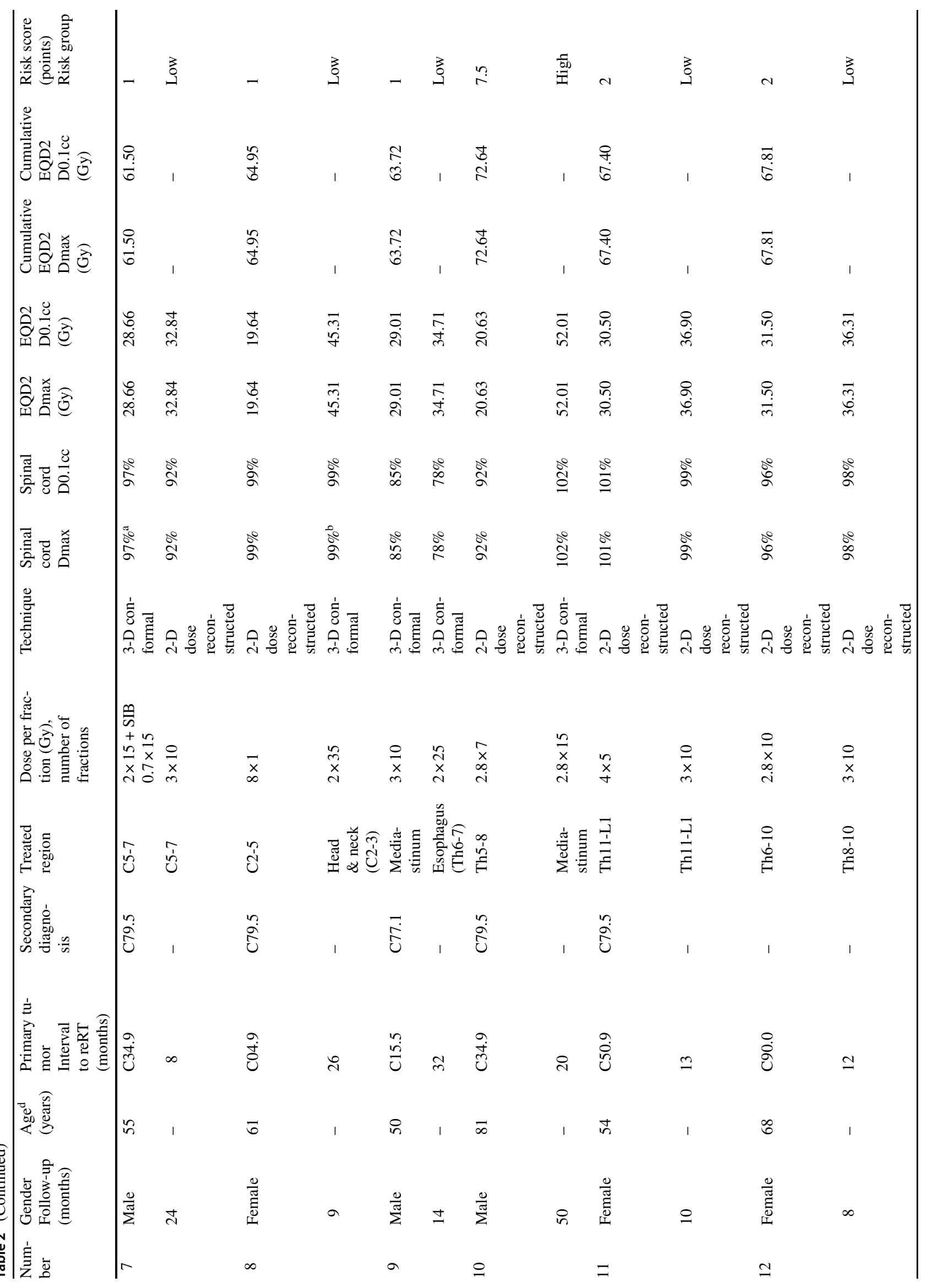




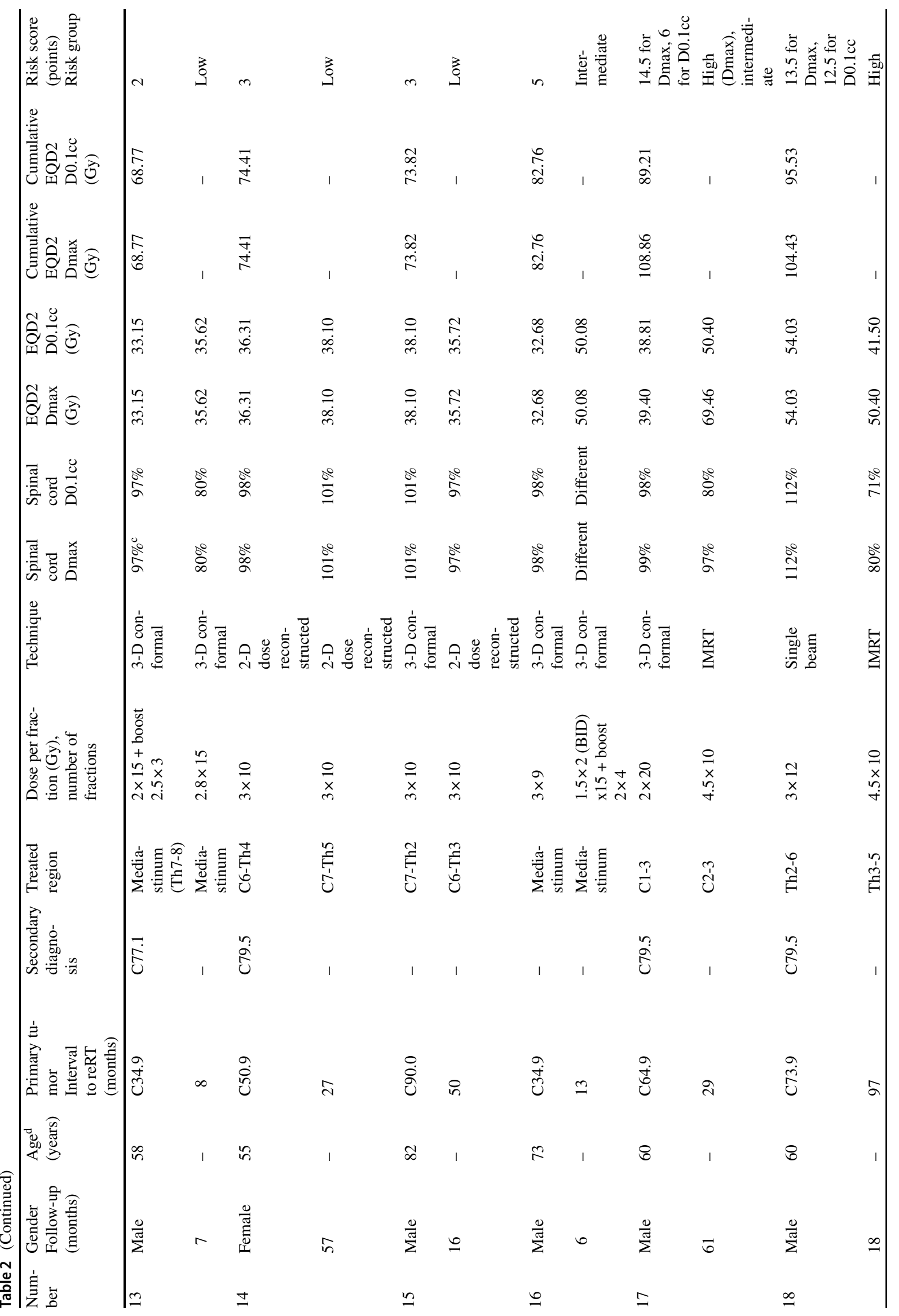




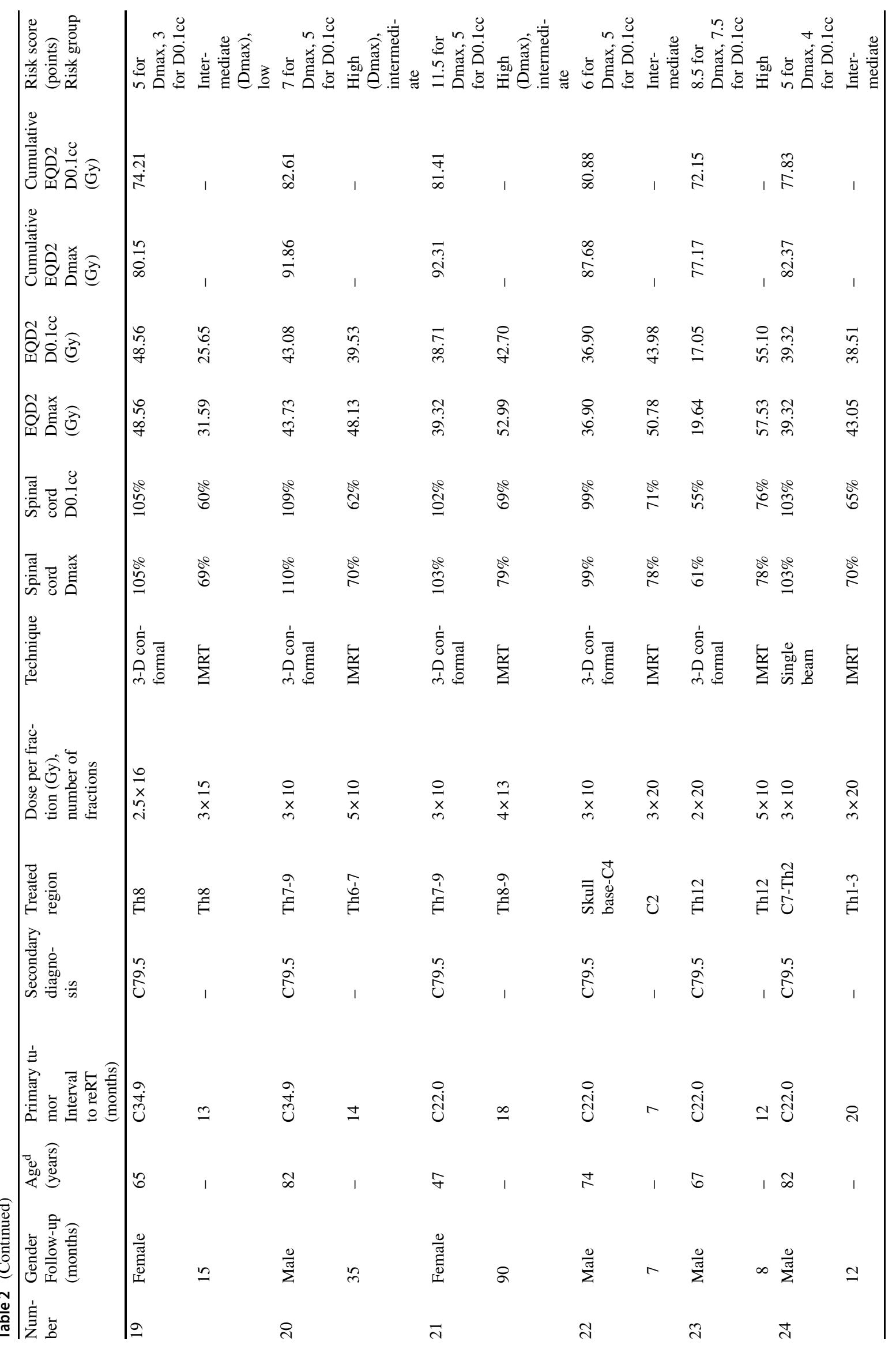




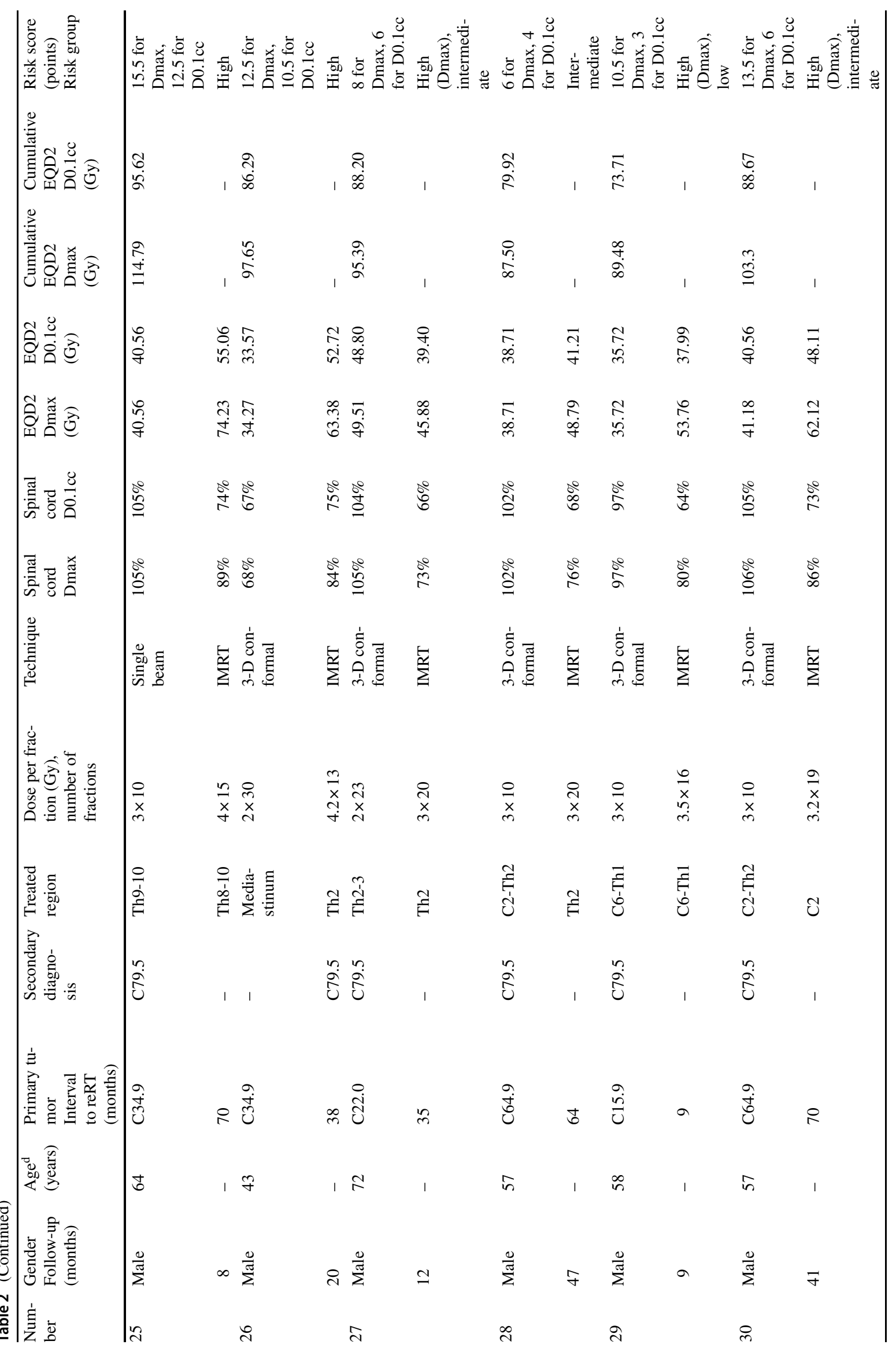




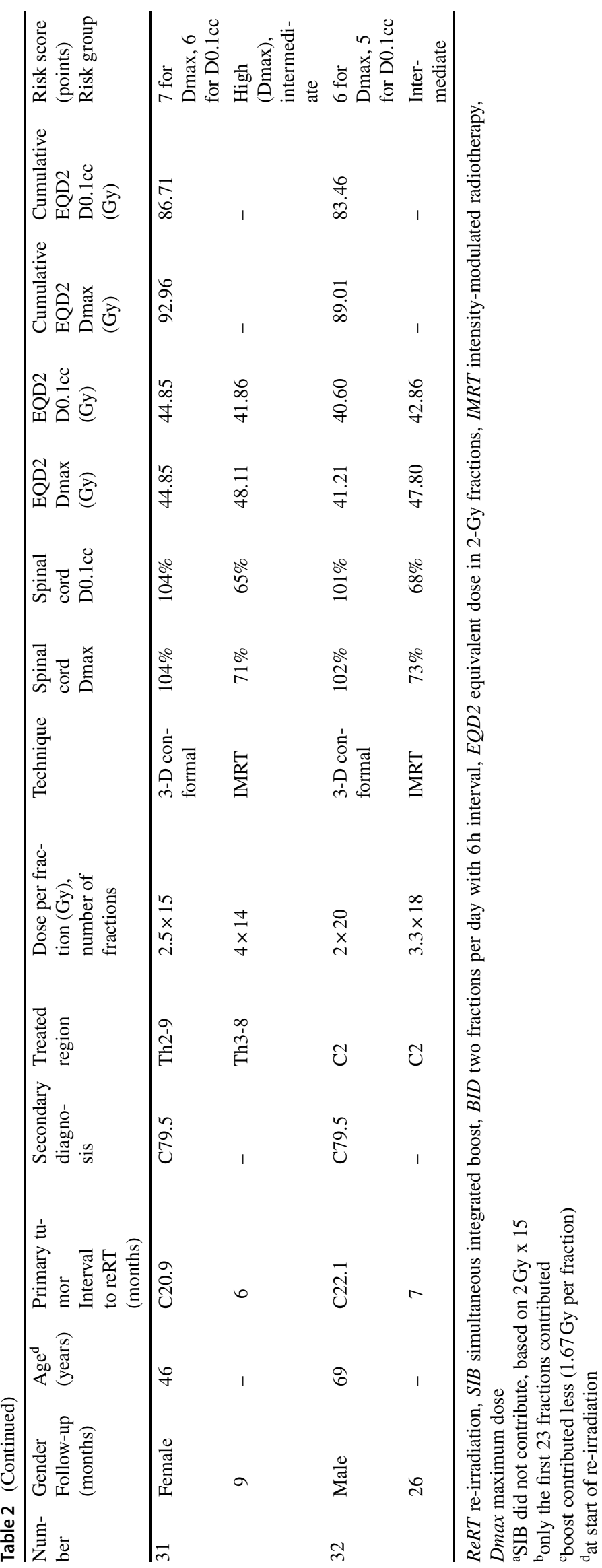


than that of the first treatment course. In most cases, Dmax was similar to D0.1cc (difference within 5\%); however, in 15 of 64 treatment courses $(23 \%)$ larger differences were registered, in line with the fact that many Japanese patients received cord-sparing intensity-modulated radiotherapy (IMRT), whereas the Norwegian center utilized simpler techniques with more homogeneous doses throughout the entire spinal canal.

The median cumulative spinal cord Dmax EQD2 was 80.7 Gy, minimum 61.12 Gy, maximum 114.79 Gy. The median cumulative spinal cord D0.1cc EQD2 was $76.1 \mathrm{~Gy}$, minimum 61.12 Gy, maximum 95.62 Gy. Besides cumulative dose, other risk factors were present (single-course Dmax EQD2 $\geq 51 \mathrm{~Gy}$ in 9 patients, single-course D0.1cc EQD2 $\geq 51$ Gy in 5 patients). The risk score (Table 1) was calculated both for Dmax and D0.1cc. For Dmax, 12 patients $(37.5 \%)$ were low risk, $8(25 \%)$ intermediate risk, and $12(37.5 \%)$ high risk. For D0.1cc, 14 patients (44\%) were low risk, $13(41 \%)$ intermediate risk, and $5(16 \%)$ high risk.

\section{Discussion}

In contrast to previous publications that mainly included dosimetric data from the two-dimensional era without MRIbased spinal cord contouring [12, 13, 17], the present biinstitutional study attempted to assess the "true" spinal cord Dmax and D0.1cc. If the actual treatment planning did not include MRI and/or 3D dose-volume histograms, the respective plans were calculated by the authors in the context of this study. Ideally, the true dose-volume histograms would form the basis of future recommendations. Our group limited inclusion to patients re-irradiated to the cervical or thoracic spine. As already discussed by Sahgal et al. [18], the Dmax has a high degree of dose uncertainty, and therefore other dose-volume histogram parameters should also be analyzed. In a previous seminal paper, Sahgal et al. compared five cases of re-irradiationinduced myelopathy to a control group of 14 re-irradiated patients with 16 spinal segments treated [19]. In the small myelopathy cohort, the median EQD2 Dmax for the SBRT component and cumulative EQD2 were 61.7Gy (range, 44.1-104.9Gy) and 99.6 Gy (range, 77.2-154.9Gy), respectively ( $\alpha / \beta$-value $2 \mathrm{~Gy}$ ). In the cohort without myelopathy, the median EQD2 Dmax for the SBRT component and cumulative EQD2 were $12.5 \mathrm{~Gy}$ (range 1.9-58.7 Gy) and 52.4Gy (range 39.1-111.2Gy), respectively. For reirradiation SBRT delivered in 1 to 5 fractions, Sahgal et al. have recommended that the cumulative thecal sac EQD2 Dmax should not exceed 70Gy [19]. According to the older risk score, $75 \mathrm{~Gy}$ to the thecal sac would still result in a low risk of myelopathy, as long as the time interval is $\geq 6$ months and neither of the two courses results in a single-course EQD2 $\geq 51$ Gy $[12,13]$.

Both institutions reporting the present data chose to exceed previous recommendations in selected cases where the administration of lower doses to the target volume was not desirable (lack of local control) and where better cord sparing could not be achieved. Of course, such individual decisions require appropriate informed consent from the patients. Fortunately, radiation myelopathy has not been observed after re-irradiation courses that were considered intermediate or high risk according to the risk score displayed in Table 1 [12, 13]. For the low-risk group, the risk of myelopathy was indistinguishable from that of first-line radiotherapy in the previous reports $(<5 \%)[12,13]$ and zero in the present study, which thereby validates the original findings. If one puts aside the methodological differences between the previous and the present reports, and chooses to add the new intermediate-risk patients to the 8 previous patients (2/8 had radiation myelopathy for a risk of $25 \%$ ), the new risk estimate would read 2/16 (based on Dmax) and $2 / 21$ (based on D0.1cc), respectively. The resulting risks of 12.5 and $9.5 \%$, respectively, would then appear lower than previously estimated. We feel that the new results lend support to the authors' current clinical practice of loosening the dose constraints for spinal cord re-irradiation if lower doses cannot be achieved, despite the limited number of patients eligible for this study. Importantly, all excluded patients who died within 6 months from re-irradiation or have shorter, ongoing follow-up also remained free from myelopathy. This finding strengthens our current policy and leads us to believe that we are not gambling with patient safety. The pros and cons of a conservative, low-myelopathy-risk dose prescription and a possibly slightly more risky "prioritize local control" prescription that involves higher doses must be explained to the patients in sufficient detail. In the Japanese re-irradiation study, which also included patients with short follow-up and/or lumbosacral re-irradiation, the 3-year local control rate was $84 \%$ [14]. This figure is in line with other results in the literature [4].

In clinical routine, different treatment planning and delivery techniques should be considered when preparing a new patient for treatment to make sure one avoids unnecessary risks, e.g., by achieving steep dose gradients so that only a small volume of the spinal cord receives a high cumulative dose. It is also necessary to minimize the risk of geographical miss, ensuring that high-dose areas do not move in unintended ways [20]. Despite several advantages of the present study compared to its predecessors, limitations must also be considered. We acknowledge that several of these are present, including the retrospective design, the limited number of eligible participants, their heterogeneous baseline and treatment characteristics, and the uncertainty of reconstructed dose distributions. Most re- 
irradiation courses employed moderate hypofractionation rather than typical SBRT fractionation. Of course, patients whose follow-up is still ongoing may be at risk of radiation myelopathy at later timepoints. Despite several experimental approaches, this severe complication, which may still be observed in the clinic [21], is difficult to modulate pharmacologically [22-24]. Hopefully, our study will encourage other institutions to publish their experiences with spinal re-irradiation, because further research is needed to confirm the limited tolerance data.

\section{Conclusion}

Even patients treated to higher cumulative doses than previously recommended, or at considerable risk of myelopathy according to a published risk score, remained free from this complication, although one must acknowledge the potential for subsequent manifestation of damage in patients currently alive, i.e., still at risk while being followed. Individualized decisions to re-irradiate after appropriate informed consent are an acceptable strategy, including scenarios where low re-irradiation doses to the spinal cord would compromise target coverage and tumor control probability to an unacceptable degree.

Author Contribution $\mathrm{CN}$ participated in the conception and design; the acquisition, analysis, and interpretation of the data; and drafted the manuscript. HD, KT, and RJO participated in the acquisition, analysis, and interpretation of the data, and helped draft the manuscript. All authors read and approved the final manuscript.

Funding Open access funding provided by UiT The Arctic University of Norway (incl University Hospital of North Norway).

Conflict of interest H. Doi, K. Tamari, R.-J. Oh, and C. Nieder declare that they have no competing interests.

Open Access This article is licensed under a Creative Commons Attribution 4.0 International License, which permits use, sharing, adaptation, distribution and reproduction in any medium or format, as long as you give appropriate credit to the original author(s) and the source, provide a link to the Creative Commons licence, and indicate if changes were made. The images or other third party material in this article are included in the article's Creative Commons licence, unless indicated otherwise in a credit line to the material. If material is not included in the article's Creative Commons licence and your intended use is not permitted by statutory regulation or exceeds the permitted use, you will need to obtain permission directly from the copyright holder. To view a copy of this licence, visit http://creativecommons.org/licenses/by/4. $0 /$.

\section{References}

1. Medin PM, Foster RD, van der Kogel AJ et al (2012) Spinal cord tolerance to reirradiation with single-fraction radiosurgery: a swine model. Int J Radiat Oncol Biol Phys 83:1031-1037
2. Ang KK, Jiang GL, Feng Y et al (2001) Extent and kinetics of recovery of occult spinal cord injury. Int J Radiat Oncol Biol Phys 50:1013-1020

3. Folkert MR, Bilsky MH, Cohen GN et al (2013) Intraoperative and percutaneous iridium-192 high-dose-rate brachytherapy for previously irradiated lesions of the spine. Brachytherapy 12:449-456

4. Myrehaug S, Sahgal A, Hayashi M et al (2017) Reirradiation spine stereotactic body radiation therapy for spinal metastases: systematic review. J Neurosurg Spine 27:428-435

5. Zschaeck S, Wust P, Graf R et al (2017) Spinal cord constraints in the era of high-precision radiotherapy: retrospective analysis of $62 \mathrm{spinal} /$ paraspinal lesions with possible infringements of spinal cord constraints within a minimal volume. Strahlenther Onkol 193:561-569

6. Mantel F, Glatz S, Toussaint A et al (2014) Long-term safety and efficacy of fractionated stereotactic body radiation therapy for spinal metastases. Strahlenther Onkol 190:1141-1148

7. Seidensaal K, Harrabi SB, Uhl M et al (2020) Re-irradiation with protons or heavy ions with focus on head and neck, skull base and brain malignancies. Br J Radiol 93:20190516

8. Simone CB 2nd, Plastaras JP, Jabbour SK et al (2020) Proton reirradiation: expert recommendations for reducing toxicities and offering new chances of cure in patients with challenging recurrence malignancies. Semin Radiat Oncol 30:253-261

9. Nieder C, Langendijk JA, Guckenberger M et al (2017) Preserving the legacy of reirradiation: a narrative review of historical publications. Adv Radiat Oncol 2:176-182

10. Nieder C, Gaspar LE, Ruysscher D et al (2018) Repeat reirradiation of the spinal cord: multi-national expert treatment recommendations. Strahlenther Onkol 194:365-374

11. Grosu AL, Andratschke N, Nieder C et al (2002) Retreatment of the spinal cord with palliative radiotherapy. Int J Radiat Oncol Biol Phys 52:1288-1292

12. Nieder C, Grosu AL, Andratschke NH, Molls M (2005) Proposal of human spinal cord reirradiation dose based on collection of data from 40 patients. Int J Radiat Oncol Biol Phys 61:851-855

13. Nieder C, Grosu AL, Andratschke NH, Molls M (2006) Update of human spinal cord reirradiation tolerance based on additional data from 38 patients. Int J Radiat Oncol Biol Phys 66:1446-1449

14. Doi H, Tamari K, Masai N et al (2021) Intensity-modulated radiation therapy administered to a previously irradiated spine is effective and well-tolerated. Clin Transl Oncol 23:229-239

15. Fowler JF, Bentzen SM, Bond SJ et al (2000) Clinical radiation doses for spinal cord: the 1998 international questionnaire. Radiother Oncol 55:295-300

16. Jones B, Hopewell JW (2019) Spinal cord re-treatments using photon and proton based radiotherapy: LQ-derived tolerance doses. Phys Med 64:304-310

17. Wong CS, Van Dyk J, Milosevic M et al (1994) Radiation myelopathy following single courses of radiotherapy and retreatment. Int $\mathbf{J}$ Radiat Oncol Biol Phys 30:575-581

18. Sahgal A, Chang JH, Ma L et al (2019) Spinal cord dose tolerance to stereotactic body radiation therapy. Int J Radiat Oncol Biol Phys. https://doi.org/10.1016/j.ijrobp.2019.09.038

19. Sahgal A, Ma L, Weinberg V et al (2012) Reirradiation human spinal cord tolerance for stereotactic body radiotherapy. Int J Radiat Oncol Biol Phys 82:107-116

20. Caravatta L, Fiorica F, Rosa C et al (2020) Role of upper abdominal reirradiation for gastrointestinal malignancies: a systematic review of cumulative dose, toxicity, and outcomes on behalf of the Re-Irradiation Working Group of the Italian Association of Radiotherapy and Clinical Oncology (AIRO). Strahlenther Onkol 196:1-14

21. Suppli MH, Munck Af Rosenschöld P, Pappot H et al (2019) Diabetes increases the risk of serious adverse events after re-irradiation of the spine. Radiother Oncol 136:130-135 
22. Wong CS, Fehlings MG, Sahgal A (2015) Pathobiology of radiation myelopathy and strategies to mitigate injury. Spinal Cord 53:574-580

23. Saager M, Hahn EW, Peschke P et al (2020) Ramipril reduces incidence and prolongates latency time of radiation-induced rat myelopathy after photon and carbon ion irradiation. J Radiat Res 61:791-798

24. Schaub SK, Tseng YD, Chang EL et al (2019) Strategies to mitigate toxicities from stereotactic body radiation therapy for spine metastases. Neurosurgery 85:729-740 